DEMOGRAPHIC RESEARCH

VOLUME 36, ARTICLE 31, PAGES 893-904 PUBLISHED 22 MARCH 2017

http://www.demographic-research.org/Volumes/Vol36/31/

DOI: 10.4054/DemRes.2017.36.31

Research Material

\title{
Extracting and reshaping World Fertility \\ Survey data in Stata
}

\section{Germán Rodríguez}

\section{Trevor Croft}

(C) 2017 Germán Rodríguez \& Trevor Croft.

This open-access work is published under the terms of the Creative Commons Attribution NonCommercial License 2.0 Germany, which permits use, reproduction \& distribution in any medium for non-commercial purposes, provided the original author(s) and source are given credit. See http://creativecommons.org/licenses/by-nc/2.0/de/ 


\section{Contents}

1 Introduction $\quad 894$

$2 \quad$ The wfs command 894

3 Viewing surveys $\quad 895$

$4 \quad$ Viewing dictionaries $\quad 896$

5
Extracting data

6 Reshaping the histories 898

$7 \quad$ Local copies 901

$8 \quad$ How it works $\quad 901$

$9 \quad$ Acknowledgments 902

$\begin{array}{lr}\text { References } & 903\end{array}$ 


\title{
Extracting and reshaping World Fertility Survey data in Stata
}

\author{
Germán Rodríguez ${ }^{1}$ \\ Trevor Croft $^{2}$
}

\begin{abstract}

\section{BACKGROUND}

The Demographic and Health Surveys (DHS) program has made available online a large number of public-use files from its predecessor, the World Fertility Survey (WFS) program, see wfs.dhsprogram.com.
\end{abstract}

\section{CONTRIBUTION}

To encourage and facilitate the use of this data, we provide a Stata command that can be used to extract and reshape the data, using local copies or working directly with the DHS data archive pages.

\footnotetext{
${ }^{1}$ Princeton University, USA. E-Mail: grodri@ princeton.edu.

2 The DHS Program, ICF International, USA.
} 


\section{Introduction}

The World Fertility Survey (WFS) program, the forerunner to the Demographic and Health Surveys (DHS) program, was a groundbreaking demographic data collection effort that conducted over 40 surveys between 1972 and 1984. To ensure that WFS's valuable datasets continue to be available to the research community, the DHS program has published a large number of public-use data files through its data archive. Please visit wfs.dhsprogram.com for a list of the surveys available.

This paper introduces a Stata command that facilitates access to WFS data, with a user-friendly syntax that allows one to view the documentation, extract a set of variables from a survey, reshape the union or birth histories from wide to long format or vice versa, and make local copies of the data and dictionary files, although users also have the option of working directly from the DHS data archive.

A pioneering feature of the WFS program was its use of machine-readable data dictionaries to document the data files. This allows our command to read the dictionaries as well as the data and to produce self-documented fully labeled Stata datasets.

By automating WFS data extraction, our command is a substantial time saver, but this does not exempt users from familiarizing themselves with the studies, recognizing that there are variations in survey design, questionnaire contents, and recoding of variables, that should inform the analysis of the data.

\section{The wfs command}

To install the command from net-aware Stata, type

net from http://wfs.dhsprogram.com

and click on the wfs link, or simply type

net install wfs, from(http://wfs.dhsprogram.com)

This will install the command and its help file.

The syntax of the command has five variants that address increasing functionality, from viewing to data extraction to reshaping the histories as well as making local copies of files. For ease of reference we provide below a syntax diagram and a brief description of each variant, followed by a more detailed explanation including examples in sections 3 to 7. In describing the syntax we follow Stata conventions, using a roman font for keywords, italics for user-specified elements, square brackets for options, and underline for minimal abbreviations. 
1. wfs

will open the home page of the WFS section of the DHS data archive using your default web browser. From there you can navigate to the country pages, as discussed in section 3 .

2. wfs using filename [, dhs]

will open the data dictionary for a given dataset using Stata's viewer, examples follow in section 4. The file name or dataset name should not include an extension. The dhs option reads the data dictionary from the DHS archive; otherwise it assumes you have a local copy.

3. wfs varlist using filename [, dhs clear]

will extract a set of variables from the specified dataset. The varlist follows Stata conventions, as explained in more detail in section 5 below. The dhs option is the same as syntax 2. The clear option overwrites the dataset in memory, if any.

4. wfs reshape long|wide [, births|unions nodrop ] will reshape the WFS data in memory from wide to long format or vice versa using the birth or union histories, as further explained in section 6 below.

5. wfs copy filename [, directory (folder) replace] will make a local copy of the data and dictionary for a given dataset, saving it in the specified folder or the current working directory. The replace option will overwrite existing files. Examples follow in section 7 below.

\section{Viewing surveys}

When you visit the WFS section of the DHS data archive, you will see links to 43 surveys from 42 countries. (The Dominican Republic had WFS surveys in 1975 and 1980.) If you click on the link for a country and year, you will see a description of the survey. For example, the page for Colombia, 1976, is at wfs.dhsprogram.com/index.cfm?ccode $=$ co.

The country pages always include a table listing all public-use files. These files are available in two different formats: WFS, a plain text ASCII format, and ISSA, a binary format that requires the ISSA (Integrated System for Survey Analysis) program, an old DOS application that is no longer available. Our command works with files in WFS format.

You will find individual standard recode data and dictionary files, such as cosr02. dat and cosr02. det for Colombia. You will also find household data and dictionaries, such as vehh02. dat and vehh02.dct for Venezuela, as well as household member data and dictionaries, such as bdhm01. dat and bdhm01.det for Bangladesh. Many countries also have supplemental standard files with additional variables, such as myss 02 . dat and myss 02 . det for Malaysia, and some have commu- 
nity data files, such a egcd0 4 . dat and egcd0 4 . det for Egypt. The files are named following the scheme ccftvn, where $\mathrm{cc}$ is a country code, $\mathrm{ft}$ is a file type, and $\mathrm{vn}$ is a version number, with extensions . det for the dictionary and . dat for the data. These are the files we can work with.

\section{Viewing dictionaries}

To view a data dictionary, you use the syntax

wfs using filename [, dhs]

where the file name omits the dictionary extension. For example, wfs using cosr02, dhs will show the machine-readable dictionary for the Colombia Standard Recode as stored in the WFS section of the DHS data archive.

Variables are typically grouped in sections. The individual standard recode files, for example, include sampling information and key dates, a union history, nuptiality variables, a birth history, fertility variables, breastfeeding, exposure status, fertility preferences, knowledge and use of contraception, background characteristics of the woman and the husband, and interview data.

The woman-level variables have names starting with $\mathrm{V}$ and a three-digit number, with the hundreds representing the content group. For example, all background variables have numbers in the 700s, and V702 is 'Type of place of residence.'

The union histories include data for up to eight unions per woman and have names starting with $\mathrm{M}$, followed by a two-digit union number and a third digit for the variable itself. For example, M012 is the date of the first union and M022 the date of the second union.

Birth histories include data for up to 24 births per woman using a similar naming scheme starting with B, so B012 is the date of the first birth and B242 the date of the 24th birth.

Some surveys also describe the union and birth histories using a compact table style with its own names. For example, BDAT refers to the dates of birth in B012 to B2 42, in addition to the detailed list. Our command, however, uses the individual variable names.

Specific surveys may include additional standard recode variables, which have names starting with X, as well as country-specific variables, with names that start with S. For example Colombia includes X701, 'Always lived in this locality,' and S107, 'Marital status $<5$ groups $>$.'

All variables have labels, and most also have value labels. For example, V702, 'Type of place of residence,' is coded 1 for 'Urban,' 2 for 'Rural,' and 99 for 'Not stated.'

The dictionaries also specify the code used for 'not applicable,' usually a string of $8 \mathrm{~s}$, and special codes, which are all codes in excess of a specified value, often a string of 
9s used for 'not stated'. For example, V702 defines 99 as a special code, and the value label tells us that it means 'Not stated.' Variable V301 is breastfeeding in the open birth interval and defines all codes above 96 as special; the value labels tell us 96 is 'Still breastfeeding', 98 is 'Did not breastfeed' and 99 is 'Not stated.'

For more detailed documentation on these data files, please refer to the WFS Data Processing Guidelines (WFS Central Staff 1980).

\section{Extracting data}

Once you have selected the variables you are interested in, the next step is to extract them, which you do using the syntax

wfs varlist using filename[, dhs]

The varlist is simply a list of the variables you want to extract. Here we follow Stata conventions and refer to all variables using lowercase names, even though they have uppercase names in the dictionary. If you wanted age and type of place of residence from the Colombian Standard Recode file, for example, you would type the command wfs v010 v702 using cosr02, dhs.

The variable list may also use

- a hyphen to refer to consecutive variables in the dictionary. For example, you may refer to v701-v705 to get the five residence and education variables.

- wildcards $\star$ and ? with the same meaning as in Stata, so $\mathrm{V} 7 \star$ will extract all variables in section 7 and $m$ ? ? 2 will extract the date of union for all unions.

- births to refer to all variables in the birth history, unions to refer to all variables in the union history, and all to extract all variables in the dictionary.

These three conventions may be freely combined in a list; for example, v010 births m??2 v701-v705 is a valid list of variables. Note, however, that wildcards may not be used to specify the beginning or end of a range, just as in Stata. And of course all only makes sense by itself.

We extract variable and value labels for all selected variables. We also recode 'not applicable' values using Stata's . n missing value. We do not recode special codes, because we think it is better for users to make their own decisions on how to handle these values.

To extract age at interview and age at first union in the Colombian survey and then tabulate age at interview, all we need is 
Rodríguez \& Croft: Extracting and reshaping World Fertility Survey data in Stata

$\begin{array}{lrrr}\text {. wfs v011 v110 using cosr02, dhs clear } & \\ \text { extracting variables v011 v110 } & \\ \text { (5378 observations read) } & & \\ \text { tab v011 } & & & \\ \text { Age }<5 \text { yr } & \text { Freq. } & \text { Percent } & \text { Cum. } \\ \text { grps> } & 1,423 & 26.46 & 26.46 \\ 15-19 & 1,051 & 19.54 & 46.00 \\ 20-24 & 842 & 15.66 & 61.66 \\ 25-29 & 599 & 11.14 & 72.80 \\ 30-34 & 579 & 10.77 & 83.56 \\ 35-39 & 476 & 8.85 & 92.41 \\ 40-44 & 408 & 7.59 & 100.00 \\ 45-49 & 5,378 & 100.00 & \\ \text { Total } & & & \end{array}$

If we tabulate age at first marriage, Stata will exclude single women because they have been coded 'not applicable':

\begin{tabular}{rrrr}
$\begin{array}{r}\text { tab v110 } \\
\text { Age at first } \\
\text { union }<7 \\
\text { grps> }\end{array}$ & Freq. & Percent & Cum. \\
\hline 15 & 348 & 10.54 & 10.54 \\
$15-17$ & 965 & 29.22 & 39.76 \\
$18-19$ & 707 & 21.41 & 61.18 \\
$20-21$ & 506 & 15.32 & 76.50 \\
$22-24$ & 419 & 12.69 & 89.19 \\
$25-29$ & 256 & 7.75 & 96.94 \\
$30+$ & 101 & 3.06 & 100.00 \\
\hline Total & 3,302 & 100.00 &
\end{tabular}

You could include single women in the table by adding the missing option (abbreviated $\mathrm{m}$ ) of the Stata tab command, which then becomes tab v110, missing.

\section{Reshaping the histories}

The union histories are stored in wide format, with one record per woman. For some purposes the analyst may need the data in long format, with one record per union. This can be done with the wfs reshape long, unions command, provided of course one has extracted the union histories. Here's an example: 


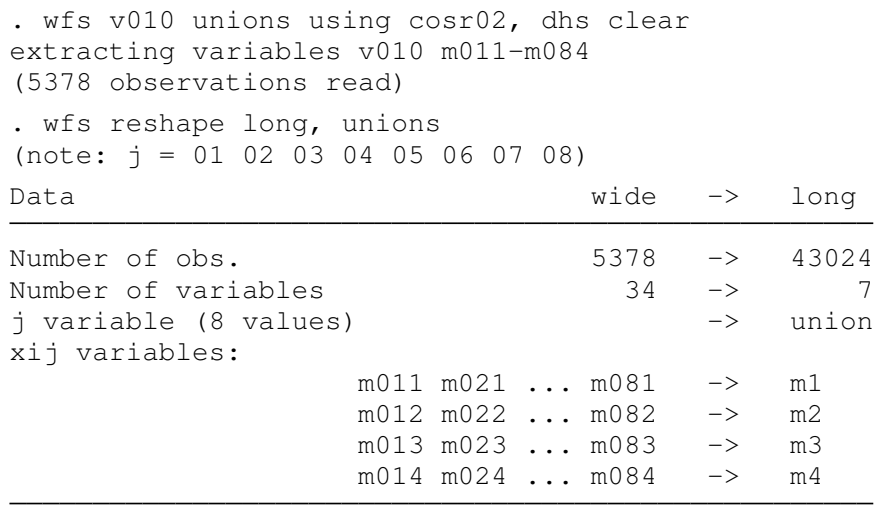

3868 unions

Our command uses Stata's own reshape command to do the bulk of the work. The Colombia dataset, which included 5,378 women, now has data for 3,868 unions. We can tabulate the type of union

\begin{tabular}{rrrr}
$\begin{array}{r}\text { tab m1 } \\
\text { Type of } \\
\text { union }<1-7>\end{array}$ & Freq. & Percent & Cum. \\
\hline $\begin{array}{r}\text { Marriage } \\
\text { Common law }\end{array}$ & 2,401 & 62.07 & 62.07 \\
\hline Total & 3,867 & 37.93 & 100.00 \\
\hline & & 100.00 &
\end{tabular}

As you can see, the wide variables m011 to m081, which have type of union for unions 1 to 8 , are now simply $\mathrm{m} 1$, and the variable and value labels have adapted to the reshape, so $\mathrm{m} 1$ is 'Type of union $<1-7\rangle$.' We also have a new variable, uni on, to record the union number.

We could use wfs reshape wide, unions to go back to a wide format with one record per woman, but of course we would then have only ever married women in the dataset, unless you specified nodrop, as explained below.

Everything said so far applies to the birth histories as well. If we extract all births, we can reshape the data to obtain one record per birth: 
Rodríguez \& Croft: Extracting and reshaping World Fertility Survey data in Stata

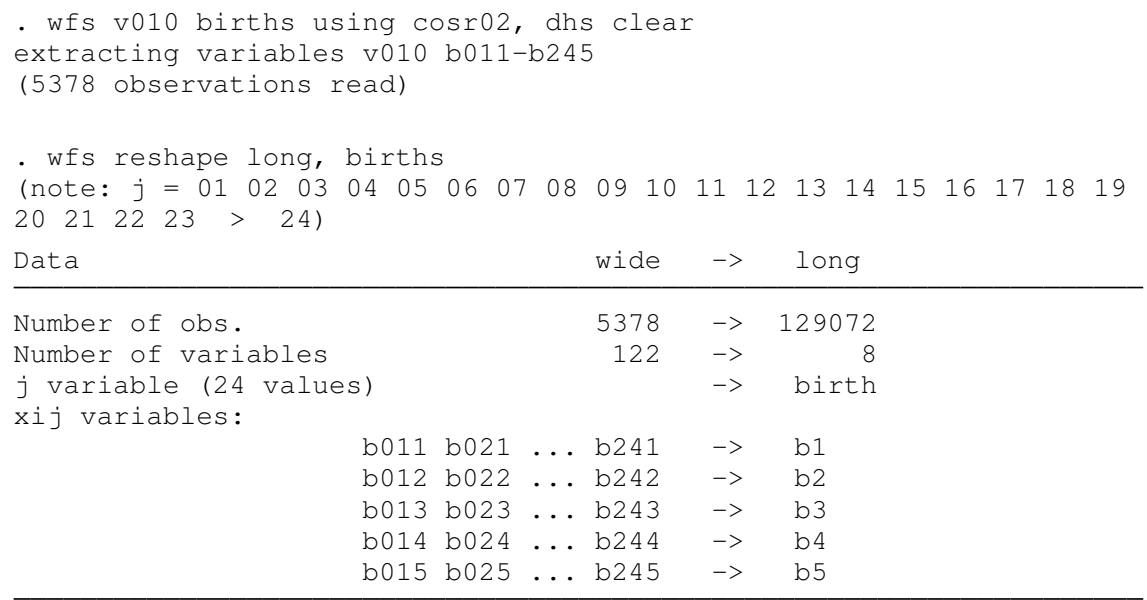

14432 births

The standard output from Stata's reshape shows that each birth variable had up to 24 instances, so we went from 5,378 women to 129,072 observations $(5,378 \times 24)$, before dropping the padding to end with 14,432 births.

The variables b015 to b245 containing the date of death (or not applicable) for each child have now become b5. We can tabulate age at death using

\begin{tabular}{rrrr}
$\begin{array}{r}\text { tab b5 } \\
\text { Age at death }<8 \\
\text { groups }>19>\end{array}$ & Freq. & Percent & Cum. \\
\hline $\begin{array}{r}<\text { Month } \\
1-2 \text { Months }\end{array}$ & 560 & 30.32 & 30.32 \\
$3-5$ Months & 151 & 8.18 & 38.49 \\
$6-11$ Months & 255 & 13.81 & 48.40 \\
1 1 Year & 286 & 15.48 & 77.69 \\
$5-9$ Years & 254 & 13.75 & 91.45 \\
$10+$ Years & 89 & 4.82 & 96.26 \\
Not stated & 52 & 2.82 & 99.08 \\
& 17 & 0.92 & 100.00 \\
\hline
\end{tabular}

to exclude surviving children, or tab b5, missing to include them. In both cases children who died but whose age at death is missing are included in the 'Not stated' category. Recall that we code 'not applicable' as missing . n but leave 'not stated' for you to handle.

The birth history may be reshaped back to wide format, but then the dataset would 
include only mothers, unless nodrop was specified. At that point one can freely go from wide to long and back to wide format as often as desired.

As noted above, when we reshape into long format, by default we keep only entries in the union or birth history that contain data, dropping empty entries which are coded 'not applicable' on all fields. The option nodrop can be used with reshape long to keep all of the entries; just type wfs reshape long, births nodrop. If you then reshape back to wide format, you will have all the original entries, including empty ones added for padding.

\section{Local copies}

A nice feature of the wfs command is that it can work directly from the DHS data archive. If you are planning to extract several subsets of variables, however, it probably pays to download the data and dictionary files to your local computer, which you can do using the syntax

$$
\text { wfs copy using filename[, directory(folder) replace] }
$$

By default, the data and dictionary files are copied to the current working directory, but the directory ( $f \circ I d e r$ ) option may be used to provide an alternative destination, provided the target directory already exists. The replace option may be used as usual to overwrite existing data and dictionary files. For example, to copy the data and dictionary for the Colombian Standard Recode file to the current working directory, you would type the command wfs copy using cosr02, replace. You would then be able to extract and reshape the data using these local copies instead of the dhs option.

\section{How it works}

The wfs command works by reading the dictionary file or downloading it if the dhs option was specified, so it can obtain information about all the variables you wish to extract. It then writes a Stata script using infix dictionary and runs it to read the data, which is downloaded by Stata if the dhs option is in effect and otherwise read from the local file system. The next step is to label the variables, create value labels, and handle any 'not applicable' codes via recodes.

The wfs reshape command uses Stata's reshape to do most of the work, generating the appropriate command, but then modifies variable and value labels to adapt to the change in the unit of analysis. The command works only if the complete union or birth histories have been downloaded. 
Rodríguez \& Croft: Extracting and reshaping World Fertility Survey data in Stata

\section{Acknowledgments}

This work has been supported by NIH grant P2CH0047879 to Princeton University and by the DHS Program funded by USAID and implemented by ICF International. 
Demographic Research: Volume 36, Article 31

\section{References}

WFS Central Staff (1980). Data processing guidelines. World Fertility Survey basic documentation 11(1-2). Voorburg: International Statistical Institute. 
Rodríguez \& Croft: Extracting and reshaping World Fertility Survey data in Stata 